This item was submitted to Loughborough's Research Repository by the author.

Items in Figshare are protected by copyright, with all rights reserved, unless otherwise indicated.

\title{
Time-volume estimation of velocity fields from nonsynchronous planar measurements using linear stochastic estimation
}

\section{PLEASE CITE THE PUBLISHED VERSION}

https://doi.org/10.1115/1.4044240

\section{PUBLISHER}

(c) American Society of Mechanical Engineers (ASME)

VERSION

AM (Accepted Manuscript)

\section{PUBLISHER STATEMENT}

This paper was accepted for publication in the journal Journal of Engineering for Gas Turbines and Power and the definitive published version is available at https://doi.org/10.1115/1.4044240.

LICENCE

CC BY 4.0

\section{REPOSITORY RECORD}

Butcher, Daniel, and Adrian Spencer. 2019. "Time-volume Estimation of Velocity Fields from Nonsynchronous Planar Measurements Using Linear Stochastic Estimation”. figshare. https://hdl.handle.net/2134/38246. 


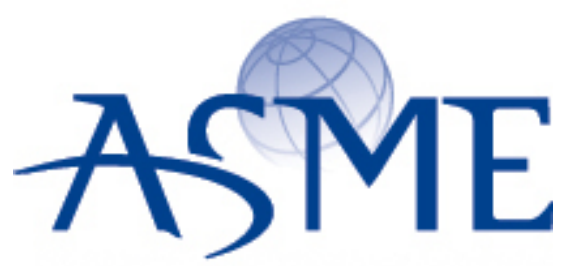

SETTING THE STANDARD

\section{American Society of Mechanical Engineers}

ASME Accepted Manuscript Repository

Institutional Repository Cover Sheet

First
Last

Time-volume estimation of velocity fields from nonsynchronous planar measurements using line ASME Paper Title: stochastic estimation

Authors: $\quad$ Daniel Butcher, Adrian Spencer

ASME Journal Title: Journal of Engineering for Gas Turbines and Power

Date of Publication (VOR* Online) _July 29,

Volume/Issue $141 / 10$

2019

https://asmedigitalcollection.asme.org/gasturbinespower/article/doi/10.1115/1.4044: ASME Digital Collection URL: Volume-Estimation-of-Velocity-Fields-From

DOI: $\quad$ https://doi.org/10.1115/1.4044240

*VOR (version of record) 


\title{
Time-Volume Estimation of Velocity Fields From Non-Synchronous Planar Measurements Using Linear Stochastic Estimation
}

\author{
Daniel Butcher ${ }^{1}$ \\ Department of Aeronautical \& Automotive Engineering, \\ Loughborough University, \\ Loughborough, \\ Leicestershire, UK, LE11 3TU \\ D.Butcher@lboro.ac.uk

\section{Adrian Spencer} \\ Department of Aeronautical \& Automotive Engineering, \\ Loughborough University, \\ Loughborough, \\ Leicestershire, UK, LE11 3TU \\ A.Spencer@lboro.ac.uk
}

\begin{abstract}
The work presented in this paper combines multiple non-synchronous planar measurements to reconstruct an estimate of a synchronous, instantaneous flow field of the whole measurement set. Temporal information is retained through the linear stochastic estimation (LSE) technique. The technique is described, applied and validated with a simplified combustor and FSN geometry flow for which 3component, 3-dimensional (3C3D) flow information is available. Using 3C3D data set, multiple virtual 'planes' may be extracted to emulate single planar PIV measurements and produce the correlations required for LSE. In
\end{abstract}

${ }^{1}$ Corresponding author 
ASME Journal of Engineering for Gas Turbines and Power

this example, multiple parallel planes are synchronised with a single perpendicular plane that intersects each of them. As the underlying data set is known it therefore can be directly compared to the estimated velocity field for validation purposes. The work shows that when the input time-resolved planar velocity measurements are first POD (proper orthogonal decomposition) filtered, high correlation between the estimations and the validation velocity volumes are possible. This results in estimated full volume velocity distributions which are available at the same time instance as the input field - i.e. a time resolved velocity estimation at the frequency of the single input plane. While 3C3D information is used in the presented work, this is necessary only for validation; in true application planar technique would be used. The study concludes that provided the number of sensors used for input LSE exceeds the number of POD modes used for pre-filtering, it is possible to achieve correlation greater than $99 \%$.

\section{INTRODUCTION}

The understanding of downstream velocity behavior is crucial to the design and development of many flow devices such as fuel injector nozzles. Great effort both in numerical modelling and experimental measurements is involved, with the state of the art in both areas becoming increasingly complex and expensive as more detailed information is sought.

The work presented in this paper will focus on the characterisation of the downstream flow of a radially-fed single stream air swirler which provides a generic swirling flow case that is applicable to fuel swirl nozzles (FSN) and combustor flows in gas turbine engines. A common approach in this application is to make use of techniques such as large eddy simulation (LES), for example, work by Dunham et. al. [2]. However, mainly due to the significant computational cost, there is an increasing use of statistical techniques. For example, Treleaven et. al. $[3,4]$ uses proper orthogonal 
decomposition (POD) to identify and study acoustic instabilities in a lean-burn FSN. This use of POD shows that not only can these techniques reduce processing time - but they may also increase the output of useful information from a data set; be it experimental or computational.

In the experimental measurement of flow fields, planar particle image velocimetry (PIV) has been commonplace for many years and it is possible to obtain high yield, high quality - both in terms of spatial and temporal resolution - data in relatively little time. Developments such as stereo-PIV (SPIV) have further improved the usefulness of the technique by allowing measurement of all three velocity components, albeit still on a single plane; 3-component, 2-dimensional (3C2D). Herein lies the problem, the measurement of volumetric data is still challenging; with several possibilities - many of which requiring increased optical access and significant investment in additional hardware.

Extensions of the PIV technique such as holographic PIV and tomographic PIV allow 3C3D volume flow measurements to be carried out. Holographic PIV is generally considered the first true volumetric PIV technique [5-7] but its use is typically limited by its complex set-up [8] and fundamental imaging issues [9]. Spencer et. al. [1] present the application of tomographic PIV to the nearfield flow of an FSN and compare against SPIV measurements of the same condition. Whilst this allowed measurement of $64 \times 64 \times 18$ 3-component, 3-dimensional (3C3D) velocity vectors, the authors describe additional complex calibration requirements for the technique. The presented experimental configuration also requires four highspeed cameras and a high-speed laser capable of 
sufficiently illuminating a volume (rather than a sheet), positioned around a highly optically-accessible test rig. Lastly, the authors suggest that tomographic PIV requires greater smoothing of the data and evaluate an error in comparison to the SPIV measurements. Despite these difficulties, the technique is increasingly popular for those applications that afford good optical access proving the appetite for 3C3D velocity information and additional flow characteristics that may be obtained. Raffell et. al. [10] describes an overview of current 3D PIV techniques.

As computational / processing power continues to improve, particle tracking techniques are becoming more accessible to provide 3C3D velocity measurements. A growing body of literature makes use of a 3D Lagrangian particle tracking velocimetry (PTV). Examples such as shake-the-box (STB) [11,12] promises high quality 4D-PTV (spatial and time resolved) over a wide range of applications, which helps reduce some of the optical access requirements. However, as with techniques already discussed, there remains a significant hardware investment and increase in optical access requirement which may just not be possible in some applications such as gas turbine combustors.

This trade-off between representative geometry and optical access requirements is well established in the experimental community and has led to increased effort to make use of alternative applications of planar PIV measurements. Scanning PIV [13] can provide a quasi 3C3D measurement using a light sheet which is stepped through the depth of the volume in time-steps which are sufficiently smaller than the PIV interframe 
time (i.e. volume sample frequency). More recently this type of system has been used to generate a moving reference plane allowing the study of vortex dynamics [14].

An alternative approach has been to make use of statistical techniques and multi-plane (asynchronous) 2(or3)C2D measurements to extract information or even reconstruct a 'measurement' volume. Volpe et. al. [15] demonstrate how a series of ten intersecting planes arranged five vertically and five horizontally may be used to study the wake topology behind a square-back Ahmed body (applicable to the automotive external aerodynamics). To understand the bi-stable wake behind the body, the temporal average of each stable state is considered by calculating two conditional averages. These are informed by a pressure signal that indicates which side of the body the wake is currently on, allowing a left-right distinction to be made. Further application of this technique is presented by Perry et. al. [16] where the authors compare the information from four 2D planes in a similar arrangement to tomographic PIV measurements at the same conditions. In that work, the authors also carry out POD analysis on the planar data to reveal bi-modal behavior; which is further revealed by investigating the tomographic PIV measurements.

In the swirling flows applicable to FSN and gas turbine combustors, the time average of planar data would not sufficiently describe the behavior of the highly threedimensional, periodic flow. Stöhr et. al. [17] and Martinelli et. al. [18] instead consider the conditional averages of the measurements according to their phase. This phase conditional averaging allows representation of the swirling flow and gives some time resolution to the reconstruction. This is an important advancement as the authors 
demonstrate the revealing of thermoacoustic oscillations that would not be possible with ensemble averaging of the planes. However, unsteady features are still not fully revealed using this approach since a phase-averaged cycle will not resolve cycle-to-cycle variations.

In conclusion, it is highly desirable to obtain time resolved volumetric velocity measurements (3C3D). Whilst techniques do exist and are fast producing high-yield, high-quality measurements, the application of these is still restricted to sufficiently optically accessible test rigs and require significant hardware investment over more traditional PIV methods. There exist a handful of statistical techniques that allow a quasi-3C3D reconstruction, some including phase information. But these do not provide a true temporal resolution.

The work presented in this paper introduces the statistical technique, linear stochastic estimation (LSE), to application of volumetric velocity reconstruction using time resolved planar PIV (3C2D) as an input. It is an improvement over phase averaging as it does not require an explicit phase indicator on which to conditionally average the data. In this way LSE offers a way to improve on understanding the cyclic variation of rotating structures rather than recreating a single cycle average and importantly it is not limited to rotating quasi-periodic flows.

\section{LINEAR STOCHASTIC ESTIMATION}

The technique of stochastic estimation, introduced by Adrian [19-21], proposes that the velocity at one location can be stochastically estimated from conditionally correlated data at an unconditional source at the same instance in time, i.e. two-point 
statistics. In the field of fluid mechanics, this presents a promising assistance to the experimental acquisition of velocity data and has been steadily developed in the time since its introduction by Adrian. The effect of higher order, quadratic stochastic estimation, QSE was soon after investigated, but found to give nearly identical estimations to the linear case, LSE [22,23].

Often, the sources for the estimation are from microphone (for example [24]) or pressure sensor arrays [25-28] as these measurements typically are less intrusive and can provide increased temporal resolution for a given experimental effort. However, disadvantages to the use of these typically wall-based sensors are described by Arnault et. al. [29] who show the limitations in the estimation of smaller turbulent structures which may not correlate well with these measurements. The study does however progress to show that it is possible to optimize the sensor locations to improve correlation using an algorithm developed by Muradore et. al. [30].

There are examples in literature that demonstrate the use of velocity measurements as the input sources for LSE. Work by Kerhervè et. al. [31] uses a hotwire rake with high temporal resolution of $30 \mathrm{kHz}$ correlated with high spatial resolution, low temporal resolution PIV measurements, generating a high spatial and temporal resolution estimation driven by the hot-wire.

The LSE technique is described mathematically by Equations 1 and 2 where $u$ is velocity, p represents the sensors (or sources), where there are NR sensors and a is the correlation matrix. This terminology will be used through the presented work.

$$
\left\langle u(x, t) p_{q}(t)\right\rangle=\sum_{r=1}^{N_{R}} a_{r}(x)\left\langle p_{r}(t) p_{q}(t)\right\rangle
$$




$$
u_{e s t}(x, t)=\sum_{r=1}^{N_{R}} a_{r}(x) p_{r}(t)
$$

Equations 1 and 2 represent the two steps required for the LSE technique. Firstly, using a data set (known as the slave) where both velocity at the desired locations and the sensor values are known simultaneously are used to generate the correlation matrix, a. For the next step, using the generated correlation matrix, only the values at each sensor location are required with Equation 2 to generate the estimation of the entire velocity field. If one considers this application to a two-dimensional velocity field initially; the slave set may be provided by a single 3C2D SPIV measurement i.e. the XY plane. The sensors may be taken as a single line across that plane, i.e. ( $X=$ const). As the line of sensor points is taken directly from the plane measurement it satisfies the requirement outlined by Adrian [19] of being at the same instance in time. Once the correlation matrix is generated, the XY plane may be subsequently estimated using a line measurement at the $\mathrm{X}=$ const line used for the correlation. In summary, Equation 1 is used to determine $a_{r}(x)$ across the slave field as the only unknown, then equation 2 uses this slave $a_{r}(x)$ matrix with sensor information $p_{r}(t)$ from the master plane to provide an estimated velocity field in the slave plane which is most likely to exist given the instantaneous state of the master plane indicated by the sensor points taken from the line of intersection.

This approach may be further extended into the 3rd spatial dimension to generate an estimation of 3C3D velocity. Figure 1 shows the $u_{z}$ velocity component contour on the YZ master plane with several example XY slave planes superimposed 
(faded). Sensors are highlighted for the upper most XY plane showing that they should lie on the intersection between each slave plane and the master plane.

The steps involved in estimation of 3C3D velocity (in the presented orientation) is then as follows:

1. Capture multiple slave $X Y$ planes (3C2D) at a range of $(Z=$ const) values of interest. These should be long time averages to ensure convergence of the 2-point statistics calculated in that plane.

2. Taking each slave plane independently, apply Equation 1. First take the multiple $\left(N_{R}\right)$ values of $p$ from along a (X=const) line, where this $\mathrm{X}$ location remains consistent between planes. Knowing $u(x)$ in this plane then allows the respective correlation matrix, $a_{r}$ to be determined as the unknown. The calculation is carried out for each velocity component independently.

3. For those velocity conditions to then be estimated using LSE, capture a single master $\mathrm{YZ}$ plane at the $(\mathrm{X}=$ const) location from the previous step. Now in this data, each $(X=$ const $)$ line may be combined with the corresponding a matrix to generate a velocity estimate in the intersecting $X Y$ plane at that value of $Z$.

Applying the technique in this manner means that all estimated velocity fields in $X Y$ planes will now be statistically synchronized to the same time instance as the master $Y Z$ plane. Any estimations of these planes may therefore be resolved at the frequency of the captured master $\mathrm{YZ}$ plane, even if this is greater than the frequency of the captured slave $\mathrm{XY}$ planes as was demonstrated by Kerhervè et. al. [31]. In fact, it is preferable to 
calculate the correlation matrix over a long time period to ensure statistical convergence.

In the application described in this work the orientation of the frames was arbitrarily chosen, but the technique could equally as well be applied with a different choice of slave / master orientation.

\section{PROPER ORTHOGONAL DECOMPOSITION}

It is quite common for the LSE technique to be used in combination with another statistical technique, POD, especially in the reconstruction of velocity fields. Also known as principle component analysis or Karhunen-Loève decomposition, POD has become widespread in the field of turbulent flow research since the 1960s [32]. It is generally used for the decomposition of turbulent flows into their larger, coherent motions and turbulence. As such a substantial body of literature exists, it is only briefly discussed in this paper. Equation 3 describes the snapshot variant of the POD technique [33] - where velocity fields, $\mathrm{u}$ are decomposed into a set of spatial modes, $\varphi(k)$ and temporal modes, $a^{(k)}$.

$$
u\left(x, t_{i}\right)=\sum_{k=1}^{M} a_{k}\left(t_{i}\right) \varphi_{k}(x) \quad i=1, \ldots, N
$$

It is widely accepted that the spatial early modes - when ordered by energy as is the norm - contains the coherent motions and the latter modes contain the turbulence. There exists a great number of techniques for choosing the 'cut-off' between these two sets. Many studies use a somewhat arbitrary value of $90 \%$ energy for example in the work by Graftieaux et. al. [34]. Butcher et. al. [35] shows how cross-correlation of the spatial modes may be used to identify the cut-off. In that work, the cut-off is used to 
determine which POD modes are representative of coherent motions which vary cyclically. The selection of the number of POD used for the reconstruction will have a significant impact on the 'smoothing' effect of the filtering.

Approaches to the combination of POD and LSE fall into two categories primarily based on whether LSE is applied to a POD filtered data set, or if the estimation is of the POD modes themselves. Podvin et. al. [36] demonstrates how coarse measurements of a single velocity component may be used in this manner to recreate POD modes and therefore reproduce the large-scale motions in the flow field on a finer mesh grid.

\section{POD-LSE coupling}

It is shown that when the LSE technique is applied to POD filtered data, there exists a coupling effect between the number of POD modes used for the initial filtering / reconstruction and the number of sensors used for the estimation of velocity fields [37]. They found that provided the number of independent sensors was sufficiently greater than the number of POD modes used for the filtering of the underlying velocity fields, a small error ( $<5 \%$ error vector magnitude) may be achieved. Figure 2 summarizes the relationship found between the number of sensors required for a desired maximum permissible error (up to a maximum of 100 sensors). In that work, the location of the multiple 'master points', $p$, were randomly selected rather than along a straight line. This mitigated the potential detrimental impact of selecting sensors in very close proximity because closely aligned locations of master points may not provide additional/independent statistical information. Therefore, it would follow that the work 
presented here may not follow this exact trend, given that the points selected are next to each other, as was required along the lines of intersection between the planes. Hence it is possible more sensors would be required than Figure 2 indicates.

\section{ACQUISITION OF TEST CASE DATA}

For the development of the presented LSE technique, a large set of high-quality data is required. It was decided that a previously published tomographic dataset [1] be used for this purpose for a number of reasons. While it is the intention of the presented work to demonstrate the effectiveness of the LSE technique in application to planar data; for purposes of validation it is necessary to have the actual measurement of volumetric velocity for comparison to the estimates. However, the authors explicitly state that for the purposes of the estimation, virtual planes are extracted from the tomographic PIV data set to simulate SPIV acquisition. To ensure no direct a priori information about the estimated velocity fields is used in their calculation, 1000 training snapshots of PIV data are used to calculate the correlation matrix and then only a single 'master' velocity plane is taken from an independent and subsequent 23 snapshots that are then used to estimate the velocity in the rest of that volume at those 23 time instances.

The test rig and injector are the same as previously reported by Midgley et. al. $[38,39]$ and Spencer et. al. [1] but is briefly described again here for completeness. The injector, depicted in Figure 3, is a radially-fed single stream swirl type, with a central jet which may be enabled as required - for the tests presented in this work, the jet is blanked. Tomographic PIV measurements of the nearfield flow were carried out in a 
(single phase) water flow facility (Figure 4). The use of water as the flow medium has several beneficial consequences. In all PIV (and PTV) measurements, it is the velocity of particles suspended in the flow which is measured, which should be representative of the flow, assuming no slip. In air this presents a challenge, particularly in high frequency flows where the large disparity between fluid densities of air and oil (typically used as seed in these experiments) can lead to induced errors. In the case of water, the greater density allows density matching of neutrally buoyant seed to be used. In the work presented, polymide seeding with mean diameter of $55 \mu \mathrm{m}$ is mixed with the water in a sump tank to ensure homogeneous seeding. This seeding gives good reflective signals so is tolerant of the lower laser energy densities found in tomographic PIV due to the formation of an illuminated volume from the laser rather than a relatively thin sheet.

The use of water as a test medium necessitates the change in refractive index to be considered. Therefore Figure 4 shows how water-filled prisms approximately matching the camera angles are positioned on the rig to ensure the change in refractive index occurs at a parallel surface to the lens plane.

Water is fed to the injector via a closed loop system with a constant header tank, providing a constant driving pressure on the injector. Immediately downstream of the injector, the duct diameter relative to the injector exit diameter was chosen to be representative of gas turbine combustors. A central axisymmetric blockage is positioned downstream at a distance of $160 \mathrm{~mm}$ from the injector plane as a controlled boundary condition, avoiding an overly elongated recirculation zone. The design requirements of this feature were obtained with CFD predictions. Further downstream, flow rate is 
controlled via a throttle valve restriction and is set to provide a nozzle exit Reynolds number of $7 \times 10^{4}$, where Reynolds number is defined using the swirler exit diameter. Using the geometrical exit area, a bulk mean velocity of $1.70 \mathrm{~ms}^{-1}$ is observed.

Illumination was provided by a Litron LDY303HE laser with each of the two cavities set to $1 \mathrm{kHz}$. This was directed through LaVision volume optics to provide a collimated illuminated volume of cross-section $70 \mathrm{~mm} \times 25 \mathrm{~mm}$. An aperture was applied to ensure sharp edges to the illuminated volume. Images were acquired using four Photron APX-RS CMOS highspeed cameras fitted with Nikor 105 mm macro lenses, each operating at $2 \mathrm{kHz}$ (synchronized) giving $1 \mathrm{kHz}$ of double frame PIV. The synchronization and control of all PIV equipment was via a LaVision high speed controller and LaVision DaVis software. The additional step required for the tomographic PIV vector calculation, error analysis and discussion is described in detail in [1] including a comparison to stereo PIV data and a self-calibration technique to reduce the effect of ghost particles. A summary of the PIV set-up and processing parameters is given in Table 1.

A total of 1023 time-steps were captured each with a resolution of $68 \times 64 \times 21$ velocity vectors, covering 1.023 seconds of flow, a significant number of flow time rotational periods. However, there are two areas in the corner of the recorded region where vector measurement was not achieved. The resulting average velocity magnitude are shown in Figure 5.

There were a few interesting points highlighted in previous work [1] about the flow condition. As it may be run with or without the central jet; the jet case had the effect of stabilizing the flow. Without the jet - as is the case here - a large central 
recirculation zone (CRZ) exists, demonstrating a precessing vortex core. This has the effect of inducing velocity fluctuations in this region. This unsteady - time-dependent feature should be predicted correctly by the application of the proposed technique.

Figure 6 and Figure 7 show slices of velocity magnitude with vectors displayed taken through the domain in the $\mathrm{YZ}$ and $\mathrm{XY}$ orientations respectively - each taken at mid-length. In general, these planes will be used for discussion and illustration throughout the remainder of the paper, as it is difficult to efficiently and effectively represent 3D data in print, particularly comparisons between 2 sets of 3D data. However, all calculations will be carried out on the full volume.

\section{DEFINITION OF PLANES}

Using the presented test case data, it is necessary to define which data will be used as slave fields and which will be used as the master plane (to drive the subsequent estimations). Firstly, of the 1023 PIV fields (time-steps) captured, let 1000 be used for the generation of the correlation matrices and contain slave planes. The remaining 23 fields shall not be used in the generation of the correlation matrices - and only the master plane from this data set shall be extracted, containing all of the intersection points (sensors) velocity. In addition, the remaining volume of this set shall later be used to allow comparison to the generated estimations.

\section{Slave planes}

Given the orientation depicted in Figure 1, the XY planes shall provide slave planes. The intersection line will be defined at $(x=0)$ of this plane; although it should be 
noted it would not necessarily need to be at this location if there were optical access issues for example. As the $\mathrm{XY}$ dimension is $(68 \times 64)$, the sensor line is therefore all data along the line $\left(N_{x}=\right.$ const, $\left.N_{y}=1 \ldots 64\right)$ for all slave planes.

To calculate the correlation matrix for each slave plane, the plane velocity matrix $(68 \times 64 \times 1000)$ is collapsed to a $(4352 \times 1000)$ matrix. This may then be used together with the sensor velocities for that plane (a matrix of $64 \times 1000$ ) in Equation 1 to generate the correlation matrix for that plane.

Each slave fields' correlation matrix effectively represents the correlation between each spatial location in the plane and every sensor. The dimensions of each correlation matrix are therefore equal to the total number of spatial locations in the plane by the number of sensors. Figure 8 presents an example of a spatial correlation matrix for the $U_{x}$ velocity component. There are a few noteworthy features of this plot. Firstly, one may expect that the correlation should be similar to the spatial correlation about that point, with a peak at the same location. However, there exist several peaks of this (normalized) distribution, none of which are near. This is due to the velocity field correlation being an ensemble of contributions from several sensors (64 in this case). However, if there were only one sensor used, then the distribution would mimic a spatial correlation.

For the presented example application there are a total of 21 slave planes (i.e. equal to the $Z$ dimension), each with three correlation matrices; one for each velocity component. However, in practice the reconstructions could be achieved with fewer (or 
greater) slave planes, depending on the permissible reliance on interpolation between estimated planes.

Master plane

Following the same orientation, the master plane shall be defined as a midlength (in the $\mathrm{X}$-direction) YZ plane. Each (X=const) line (examples presented in Figure 9) is taken from the master plane set and used with the appropriate correlation matrix and Equation 2 to generate the volume velocity field.

\section{ASSESSMENT OF GENERATED ESTIMATIONS AND DISCUSSION}

The process is carried out as described using the MATLAB program for each of the 23 validation master planes (time-steps) using 60 POD mode pre-filtering. Firstly, the ensemble average of the reconstruction of the estimated fields is presented in Figure 10 and can be compared to the ensemble average for the PIV data presented earlier (Figure 5).

It can be seen in Figure 10 that there are slight differences to the PIV data posted in Figure 5. In fact, the validation shows agreement between the estimation and the validation data set. Differences arise as Figure 10 is the ensemble of only the estimations from the 23 master planes extracted from the validation set; whereas Figure 5 shows the ensemble of the 1000 slave velocity fields. Further exploration of the quality of the estimation is given in the remainder of this paper.

The purpose of carrying out the LSE technique - rather than the earlier discussed ensemble average reconstructions - is to provide time-volume velocity fields. And as 
explained earlier, using fewer POD modes in the master field reconstruction than number of LSE sensors leads to less than $1 \%$ error in the estimation. The following discussion is regarding 60 POD mode reconstruction; the reader is reminded that there are a total of 64 LSE sensors at each intersection of planes, and therefore this condition is satisfied. By selecting an extract of an estimated XY plane from this data set; a direct comparison can be made to the equivalent field extracted from the PIV validation set (at the same time instance). This is presented in Figure 11a \& b respectively.

Whilst the comparison is presented in this way (Figure 11), the two fields have very small disparity between them, $<<1 \%$. This confirms the finding in literature [37] regarding POD pre-filtering.

As part of the investigation, all three velocity components, across all 23 validation fields were compared to their validation measurement counterparts. A similar finding was observed in every condition. Therefore, the study concludes that the application of this technique may effectively estimate time-volume velocities from a single master plane measurement set.

Further, an investigation is carried out to establish the level of error introduced to the estimation if the number of POD modes is increased beyond the number of LSE sensors. For this purpose, five conditions were investigated, based on the number of POD modes used for pre-filtering: 70, 100, 130, 160 and no POD pre-filtering.

Considering the first mentioned condition, 70 modes, they may be directly compared by overlaying the data-set - this was not previously possible due to there being no discernable discrepancy between the two. Figure 12 shows how now that the 
number of sensors is greater; albeit by only around $10 \%$, there is some discrepancy between the estimation and the validation data. However, note the scale change in Figure 12 required before this difference is visible - showing there is still a strong similarity between the two velocity fields. This would be expected to become more significant as less pre-filtering (i.e. more POD modes) are included in the master planes.

To efficiently assess the accuracy of the estimations, a quantitative approach is taken. Two velocity fields may be directly compared by a correlation between the two. Equation 4 gives the correlation, $\mathrm{R}$ for a velocity component i between two vector fields, $A$ and $B$. Note, there is no time component of this equation, and it is therefore applied to each of the 23 timesteps independently and the value of $\mathrm{R}$ for each condition is averaged.

$$
R_{i i, A B}=\frac{\left\langle u_{i}(x)_{A} u_{i}(x)_{B}\right\rangle}{\sqrt{\left\langle u_{i}^{2}(x)_{A}\right\rangle} \sqrt{\left\langle u_{i}^{2}(x)_{B}\right\rangle}}
$$

Each of the pre-filtered cases are estimated and the correlation between estimations and validation time-volume calculated. Correlations for these conditions are shown in Figure 13.

The correlations in Figure 13 show that reasonable estimations may be made in the cases where the number of POD modes used for pre-filtering exceeds the number of LSE sensors, but this is dependent on what the acceptable level of error is deemed to be. Also included is the condition with no POD filtering (represented by the dashed line). Even in the worst-case scenario, where there is no POD pre-filtering of the data, greater than $95 \%$ correlation exists between the estimated fields and the validation fields. It is 
suspected that while the no-filtering cases did not utilize POD, it was necessary in their capture for gaussian filtering of the data due to noise on the tomographic measurements which may have impacted this result - leading to an unexpectedly high correlation in the no POD filtering condition. A side effect of this initial filtering (applied to all data in previous works [1]) will have been to remove some of the smaller structures that would otherwise have been represented by the higher order modes. Therefore, effectively less information is required to estimate the velocity fields than may otherwise be the case.

\section{METHOD CRITIQUE AND FUTURE WORK}

This paper provides a validation of the LSE technique using a 3D-3C tomographic PIV data set where direct measurements are available to test the accuracy of the estimation results. This has been done carefully to ensure a priori knowledge available in this data set has not been used to assist the methodology. The origins, quality and uncertainty in this data has been previously described in [1]. Based on the divergence of the velocity field which should be zero, in this incompressible flow, it was estimated the instantaneous velocity uncertainty from the tomographic PIV was better than $\pm 10 \%$ to $95 \%$ confidence.

The tomographic set up required for this validation does require a high degree of optical access. However, the technique is aimed at enabling 2D planar 2or3C PIV data to be volume reconstructed on an instantaneous basis. Without such an approach only time or phase averaged statistics can be meaningfully reconstructed into a volumetric picture. The proposed LSE method is aimed for use with a standard mono or stereo PIV 
system. Thus, the optical access to employ the technique would ordinarily be commensurate with a standard mono or stereo PIV systems with sufficient flexibility to traverse the measurement plane through the volume of interest.

Alignment of the measurement planes is an issue that has not been directly addressed in this validation. For the approach to work, the line of intersection in the master and slave planes will need to be identified with some degree of accuracy, otherwise the sensors from the master and slave plane will not be at identical locations. Additionally, there may be some differences in velocity uncertainty from the two planes through alternate calibration of the PIV planes. There are solutions to these issues and is the subject of current work.

Where there is not good PIV vector yield (less than 95\% first choice vectors) then additional steps are required to ensure the LSE methodology provides mathematically robust estimates. Again, this is the subject of future work, but it would be expected that uncertainty of velocity from the stereo PIV would be reduced to typically around half of that of this tomographic PIV data, and this should help to improve the LSE results.

It is seen in this validation exercise that there can be very good correlation in the estimated and actual instantaneous velocity fields. This is unlikely to be as good if large scale coherent structures do not exist in the turbulence field. LSE can work with small scale turbulence, but sensor locations would need to be more uniformly distributed around the slave plane so that their typical spacing was not too far from the integral length scales that exist in the flow. Where sensors come from a line of intersection 
between two planes the user would not have the luxury of choosing the required sensor distribution and this application of LSE would be less valuable. However, there would be little to gain of instantaneous volume reconstruction as the flow field becomes more and more self-similar throughout, as in the case of near-homogeneous turbulence.

As a point in the estimated flow field becomes more remote from the sensor locations located on the plane of intersection then the magnitude of the 2-point correlations becomes smaller and the quality of the reconstruction would be expected to become poorer. This is demonstrated in Figure 14.

The profiles in Figure 14 are along two secant lines through a of the near circular swirl cone $(z / D s=0.02)$. They run parallel to the diametral line on which the sensor points are taken from the master plane. In Figure 14 the profile is close to the diametral line. In Figure $b$ the profile is at about a quarter diameter further away. The two peaks in the turbulent profile associated with the swirl cone and the inner shear layer helical vortex structures are thus thickened on the far field chord line as it cuts the swirl cone obliquely. In this flow where the turbulence field has a strong coherence to it, it can be seen the LSE estimation captures the flow features, and the higher order statistics associated with them, well. The quality of the second order statistic degrades as the LSE estimated flow field becomes more remote from the sensor locations. In the example above the tke is up to $40 \%$ lower using LSE directly. However, using POD filtered data prior to applying the LSE reconstruction as advocated in this paper, then in the example presented, the turbulence statistics are captured with as good fidelity as allowed by the 
POD reconstruction, where that POD reconstruction is generated by as many spatial modes as there are sensor locations available in the master plane.

\section{CONCLUSION}

A method is developed and presented allowing a time-volume velocity estimation to be generated based on a set of sensors of planar measurements. To demonstrate the methodology, it has been applied to a simplified gas turbine combustor and FSN flow for which existing time-volume velocity measurements were available to allow for effective validation. Although the full volume information was available, virtual planes have been extracted and used to emulate planar measurement inputs. The advantage of this approach is a priori knowledge of the flow field allows a direct comparison between the estimations and the measured velocities.

During the first step of the technique, akin to a calibration procedure, the spatial resolution of the final estimations is determined by the number of slave fields to be captured, and subsequent correlations generated against the master plane intersection.

The temporal resolution of the estimations is then determined by the frequency of the master plane measurements under test conditions. This highlights a potential further use of the technique; if high-speed planar information is available, then the full volume estimation will also be at this frequency.

The link between POD filtering and LSE reconstructions suggested in literature has been investigated in this application. The findings generally agree strongly, but there is some evidence that other PIV filtering techniques have smoothed the data and removed some of the smaller structures of the flow. 
ASME Journal of Engineering for Gas Turbines and Power

Finally, the application of this technique does not necessarily require new data sets, it may be retrospectively applied to any data where intersecting non-synchronised measurements have been carried out. Indeed, these may generally be velocity fields from PIV data but the mathematical approach would work well with other quantities such as pressure or scalar fields from LIF in both non-reacting or reacting flow fields. 
ASME Journal of Engineering for Gas Turbines and Power

\section{NOMENCLATURE}

\begin{tabular}{|c|c|}
\hline $3 C 3 D$ & 3-component, 3-dimensional (velocity) \\
\hline$a_{r}$ & Correlation matrix (component r) \\
\hline$C R Z$ & Central recirculation zone \\
\hline FSN & Fuel swirl nozzle \\
\hline LES & Large eddy simulation \\
\hline LSE & Linear stochastic estimation \\
\hline$p_{r}$ & Sensor ( $r)$ \\
\hline PIV & Particle image velocimetry \\
\hline$P O D$ & Proper orthogonal decomposition \\
\hline PTV & Particle tracking velocimetry \\
\hline$R_{i i, A B}$ & Correlation between fields $A$ and $B$ \\
\hline SPIV & Stereo particle image velocimetry \\
\hline STB & Shake-the-box \\
\hline$U_{\text {est }}(x, t)$ & Estimated $u$ velocity component \\
\hline
\end{tabular}




\section{REFERENCES}

[1] Spencer, A., Brend, M., Butcher, D., Dunham, D., Cheng, L., and Hollis, D., 2018, "Tomographic PIV in the Near Field of a Swirl-Stabilised Fuel Injector," Proc. ASME Turbo Expo 2018, pp. 1-10.

[2] Dunham, D., Spencer, A., Mcguirk, J. J., and Dianat, M., 2008, "Comparison of URANS and LES CFD Methodologies for Air Swirl Fuel Injectors," Proc. ASME, pp. 1-10. [3] Treleaven, N. C. W., Su, J., Garmory, A., and Page, G. J., 2017, "The Response To Incident Acoustic Waves Of The Flow Field Produced By A Multi-Passage Lean-Burn Aero-Engine Fuel Injector," Proceedings of ASME Turbo Expo 2017: Turbomachinery Technical Conference and Exposition GT2017, Charlotte, NC, USA, pp. 1-14.

[4] Treleaven, N. C. W., Su, J., Garmory, A., Page, G. J., and Juniper, M., 2018, "The Identification And Prediction Of Helical Modes Induced By A Multi-Passage Swirl Stabilised Lean Burn Aero-Engine Fuel Injector Under Steady State And Acoustically Forced Conditions," Proceedings of ASME Turbo Expo 2018 Turbomachinery Technical Conference and Exposition GT2018, Oslo, Norway, pp. 1-14.

[5] Barnhart, D. H., Adrian, R. J., and Papen, G. C., 1994, "Phase-Conjugate Holographic System for High-Resolution Particle-Image Velocimetry," Appl. Opt., 33(30), p. 7159.

[6] Meinhart, C. D., Barnhart, D. H., and Adrian, R. J., 1996, "Interrogation and Validation of Three-Dimensional Vector Fields," Developments in Laser Techniques and Applications to Fluid Mechanics, pp. 379-391.

[7] Meng, H., and Hussain, F., 1995, "In-Line Recording and off-Axis Viewing Technique for Holographic Particle Velocimetry," Appl. Opt., 34(11), p. 1827.

[8] Scherer, J. O., and Bernal, L. P., 1997, "In-Line Holographic Particle Image Velocimetry for Turbulent Flows," Appl. Opt., 36(35), pp. 9309-9318.

[9] Pu, Y., and Meng, H., 2005, "Four-Dimensional Dynamic Flow Measurement by Holographic Particle Image Velocimetry," Appl. Opt., 44(36), pp. 7697-7708.

[10] Raffel, M., Willert, C. E., Scarano, F., Kahlet, C. J., Wereley, S. T., and Kompenhams, J., 2018, "Techniques for 3D-PIV," Particle Image Velocimetry, Springer, Cham, pp. 309-365.

[11] Schanz, D., Schröder, A., and Gesemann, S., 2014, “'Shake The Box' - a 4D PTV Algorithm: Accurate and Ghostless Reconstruction of Lagrangian Tracks in Densely Seeded Flows," International Symposium on Applications of Laser Techniques to Fluid Mechanics, pp. 7-10.

[12] Schanz, D., Schröder, A., Gesemann, S., and Wieneke, B., 2015, "'Shake the Box': Lagrangian Particle Tracking in Densely Seeded Flows at High Spatial Resolution," 9th Int. Symp. Turbul. Shear Flow Phenomena, TSFP 2015, 3(Elsinga 2006).

[13] Brücker, C., 1995, "Digital-Particle-Image-Velocimetry (DPIV) in a Scanning LightSheet: 3D Starting Flow around a Short Cylinder," Exp. Fluids, 19(4), pp. 255-263.

[14] Ponitz, B., Sastuba, M., and Brücker, C., 2016, "4D Visualization Study of a Vortex Ring Life Cycle Using Modal Analyses,” J. Vis., 19(2), pp. 237-259. 
[15] Volpe, R., Devinant, P., and Kourta, A., 2015, "Experimental Characterization of the Unsteady Natural Wake of the Full-Scale Square Back Ahmed Body: Flow Bi-Stability and Spectral Analysis," Exp. Fluids, 56(5), pp. 1-22.

[16] Perry, A.-K., Almond, M., Passmore, M., and Littlewood, R., 2016, "The Study of a Bi-Stable Wake Region of a Generic Squareback Vehicle Using Tomographic PIV," SAE Int. J. Passeng. Cars - Mech. Syst., 9(2), pp. 743-753.

[17] Stohr, M., Sadanandan, R., and Meier, W., 2011, "Phase-Resolved Characterization of Vortex-Flame Interaction in a Turbulent Swirl Flame," Exp. Fluids, 51(4), pp. 1153-1167.

[18] Martinelli, F., Cozzi, F., and Coghe, A., 2012, "Phase-Locked Analysis of Velocity Fluctuations in a Turbulent Free Swirling Jet after Vortex Breakdown," Exp. Fluids, 53(2), pp. 437-449.

[19] Adrian, R. J., 1977, "On the Role of Conditional Averages in Turbulence Theory," Turbul. Liq., (JANUARY 1977), pp. 323-332.

[20] Adrian, R. J., 1979, "Conditional Eddies in Isotropic Turbulence," Phys. Fluids, 22(11), pp. 2065-2070.

[21] Adrian, R. J., 1996, "Stochastic Estimation of the Sturcture of Turbulent Fields," Eddy Structure Identification., J.P. Bonnet, ed., Springer, Vienna, pp. 145-195.

[22] Tung, T. C., and Adrian, R. J., 1980, "Higher-Order Estimates of Conditional Eddies in Isotropic Turbulence," Phys. Fluids, 23(7), pp. 1469-1470.

[23] Tinney, C. E., Coiffet, F., Delville, J., Hall, A. M., Jordan, P., and Glauser, M. N., 2006, “On Spectral Linear Stochastic Estimation," Exp. Fluids, 41(5), pp. 763-775.

[24] Gutmark, E. J., Verfaillie, S., Bonnet, J.-P., and Grinstein, F., 2006, "Linear Stochastic Estimation of a Swirling Jet," AIAA J., 44(3), pp. 457-468.

[25] Hosseini, Z., Martinuzzi, R. J., and Noack, B. R., 2015, "Sensor-Based Estimation of the Velocity in the Wake of a Low-Aspect-Ratio Pyramid," Exp. Fluids, 56(1).

[26] Taylor, J. A., and Glauser, M. N., 2004, "Towards Practical Flow Sensing and Control via POD and LSE Based Low-Dimensional Tools," J. Fluids Eng., 126(3), pp. 337345.

[27] Naguib, A. M., Wark, C. E., and Juckenhöfel, O., 2001, "Stochastic Estimation and Flow Sources Associated with Surface Pressure Events in a Turbulent Boundary Layer," Phys. Fluids, 13(9), pp. 2611-2626.

[28] Hudy, L. M., and Naguib, A., 2007, "Stochastic Estimation of a Separated-Flow Field Using Wall-Pressure-Array Measurements," Phys. Fluids, 19(2).

[29] Arnault, A., Dandois, J., Monnier, J. C., Delva, J., and Foucaut, J. M., 2016, "Analysis of the Filtering Effect of the Stochastic Estimation and Accuracy Improvement by Sensor Location Optimization," Exp. Fluids, 57(12), pp. 1-22.

[30] Muradore, R., Bezzo, F., and Barolo, M., 2006, "Optimal Sensor Location for Distributed-Sensor Systems Using Multivariate Regression," Comput. Chem. Eng., 30(3), pp. 521-534.

[31] Kerhervé, F., Roux, S., and Mathis, R., 2017, “Combining Time-Resolved MultiPoint and Spatially-Resolved Measurements for the Recovering of Very-Large-Scale Motions in High Reynolds Number Turbulent Boundary Layer," Exp. Therm. Fluid Sci., 82, pp. 102-115. 
[32] Lumley, J. L., 1967, "The Structure of Inhomogeneous Turbulent Flows," Atmos. Turbul. Radio Wave, Nauk., pp. 166-178.

[33] Sirovich, L., 1987, "Turbulence and Dynamics of Coherent Structues. Part I: Coherent Structures," Q. Appl. Math., 45(3), pp. 561-571.

[34] Graftieaux, L., Michard, M., and Grosjean, N., 2001, "Combining PIV, POD and Vortex Identification Algorithms for the Study of Unsteady Turbulent Swirling Flows," Meas. Sci. Technol. Meas. Sci. Technol, 12(1201), pp. 1422-1429.

[35] Butcher, D., Spencer, A., and Chen, R., 2018, "Influence of Asymmetric Valve Strategy on Large-Scale and Turbulent in-Cylinder Flows," Int. J. Engine Res., 19(6), pp. 631-642.

[36] Podvin, B., Nguimatsia, S., Foucaut, J. M., Cuvier, C., and Fraigneau, Y., 2018, “On Combining Linear Stochastic Estimation and Proper Orthogonal Decomposition for Flow Reconstruction," Exp. Fluids, 59(3), pp. 1-12.

[37] Butcher, D., and Spencer, A., 2019 "Linear Stochastic Estimation of Coherent Structures in Internal Combustion Engines Flow," Int. J. Engine Res.

[38] Midgley, K., Spencer, A., and McGuirk, J. J., 2005, "Unsteady Flow Structures in Radial Swirler Fed Fuel Injectors," J. Eng. Gas Turbines Power, 127(4), p. 755.

[39] Spencer, A., McGuirk, J. J., and Midgley, K., 2008, "Vortex Breakdown in Swirling Fuel Injector Flows," J. Eng. Gas Turbines Power, 130(2), p. 021503. 
ASME Journal of Engineering for Gas Turbines and Power

\section{Table Caption List}

Table $1 \quad$ Summary of experimental set-up parameters 


\section{Figure Captions List}

Fig. 1 Location of $\mathrm{YZ}$ (master) plane and examples of $\mathrm{XY}$ (slave) planes with sensors highlighted with 'o'. FSN exit at $(0,0,0)$

Fig. 2 Relationship between number of POD modes used for input velocity fields and the number of sensors required for a maximum permissible error (data from [37])

Fig. 3 Radially-fed single stream injector

Fig. $4 \quad$ Water flow test facility with tomographic PIV

Fig. 5 Velocity magnitude contour and iso-surface showing the ensemble average flow field from the captured tomographic PIV data

Fig. 6 YZ slice showing ensemble average velocity vectors from the captured tomographic PIV data $(X=0)$

Fig. $7 \quad X Y$ slice showing ensemble average velocity vectors from the captured tomographic PIV data $(Z=0.25)$

Fig. 8 Example of correlation matrix for Ux for the indicated sensor location. Correlation is normalized according to the maxima in the contour.

Fig. 9 Example of instantaneous master plane with 3 example sensor sets highlighted. Contour shows velocity magnitude and in-plane vectors are displayed 
Fig. 10 Ensemble mean of estimated velocity fields. Contours show velocity magnitude. Obtained from master fields with 60 POD mode reconstruction.

Fig. 11(a) Example extract of estimated velocity field, generated using 60 POD spatial modes

Fig. 11(b) Extract of the same plane and time instance taken from the validation PIV data set

Fig. 11 Comparison of estimated velocity and validation velocity measurements for the same time instance (validation data set, $\mathrm{t}=1010$ )

Fig. 12 Comparison between validation data and estimated field (master plane pre-filtered, 70modes)

Fig. 13 Mean correlation of estimated velocity and validation velocity for a range of pre-filtered and non-pre-filtered conditions

Fig. 14(a) Near field, $y / D s=0.03, z / D s=0.02$

Fig. 14(b) Far field, y/Ds $=0.27, z / D s=0.02$

Fig. 14 Profiles of turbulent kinetic energy on a line parallel to the line of sensors. (intersection line at, $y / D s=0.00, z / D s=0.02$ ) 
Table 1 Summary of experimental set-up parameters

\begin{tabular}{|c|c|}
\hline \multicolumn{2}{|l|}{ Seeding } \\
\hline Material & Polymide \\
\hline Mean diameter & $55 \mu \mathrm{m}$ \\
\hline \multicolumn{2}{|l|}{ Imaging } \\
\hline Lens focal length & $105 \mathrm{~mm}$ \\
\hline Resolution & 1 M.pixel \\
\hline Mounting angle & $\sim 25^{\circ}$ (relative to $\mathrm{x}$-axis) \\
\hline Interframe time, $\delta \mathrm{t}$ & $30 \mu \mathrm{s}$ \\
\hline \multicolumn{2}{|l|}{ Illumination } \\
\hline $\begin{array}{l}\text { Volume cross- } \\
\text { section }\end{array}$ & $70 \times 25 \mathrm{~mm}$ \\
\hline \multicolumn{2}{|l|}{ PIV processing } \\
\hline $\begin{array}{l}\text { Measurement } \\
\text { volume }\end{array}$ & $1000 \times 1000 \times 250$ voxel \\
\hline $\begin{array}{l}\text { Pre-processing } \\
\text { steps: }\end{array}$ & $\begin{array}{l}\text { - Sliding minimum } \\
\text { subtraction (1000 frames) } \\
\text { - } 3 \times 3 \text { gaussian smoothing } \\
\text { - Volume self-calibration }\end{array}$ \\
\hline MART iterations & $\begin{array}{l}2 \times(64 \times 64 \times 64 \text { with } 75 \% \\
\text { overlap) }\end{array}$ \\
\hline Vector resolution & $64 \times 64 \times 183 \mathrm{C} 3 \mathrm{D}$ \\
\hline Post-processing & $3 \times 3$ gaussian smoothing \\
\hline
\end{tabular}


ASME Journal of Engineering for Gas Turbines and Power

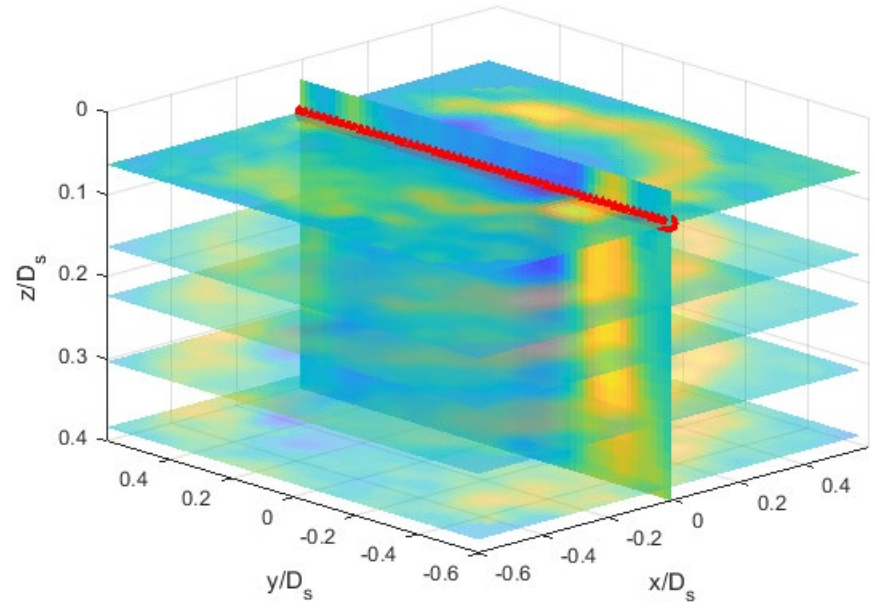

Fig. 1 Location of YZ (master) plane and examples of XY (slave) planes with sensors highlighted with 'o'. FSN exit at $(0,0,0)$ 
ASME Journal of Engineering for Gas Turbines and Power

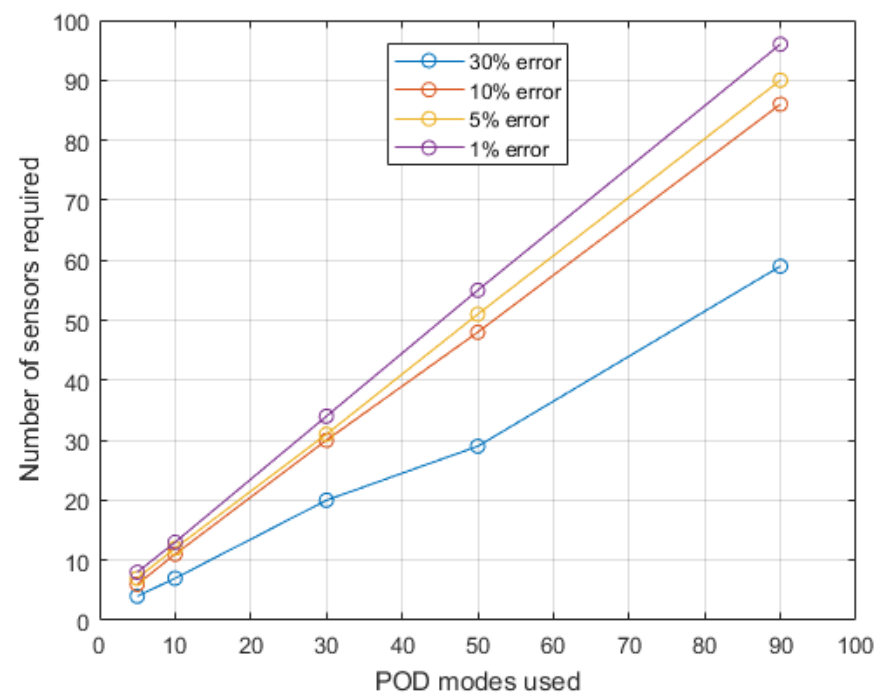

Figure 2 Relationship between number of POD modes used for input velocity fields and the number of sensors required for a maximum permissible error (data from [37]) 
ASME Journal of Engineering for Gas Turbines and Power

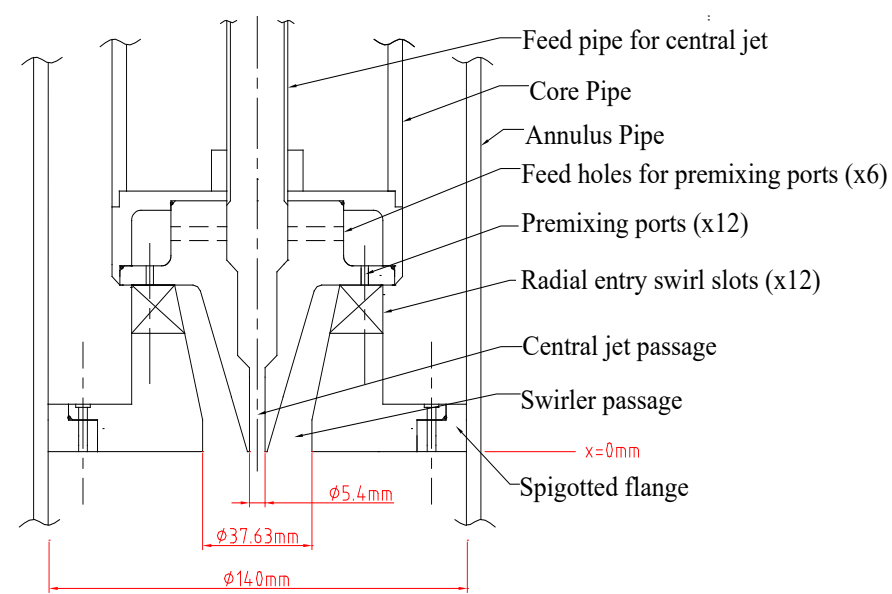

Figure 3 Radially-fed single stream injector 
ASME Journal of Engineering for Gas Turbines and Power

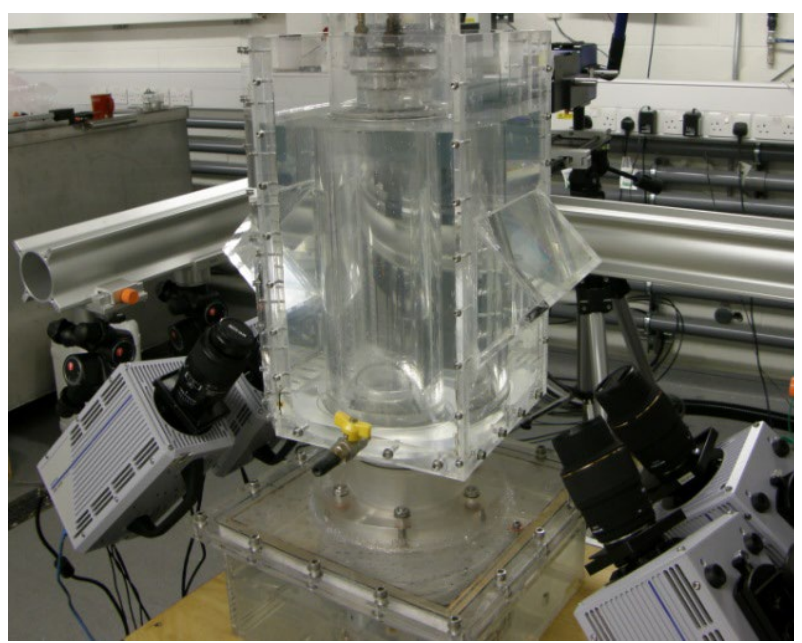

Figure 4 Water flow test facility with tomographic PIV 
ASME Journal of Engineering for Gas Turbines and Power

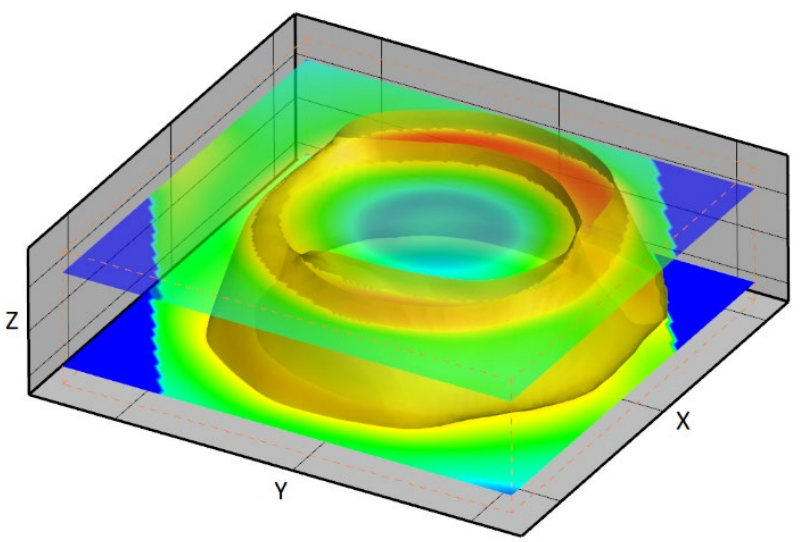

Figure 5 Velocity magnitude contour and iso-surface showing the ensemble average flow field from the captured tomographic PIV data 
ASME Journal of Engineering for Gas Turbines and Power

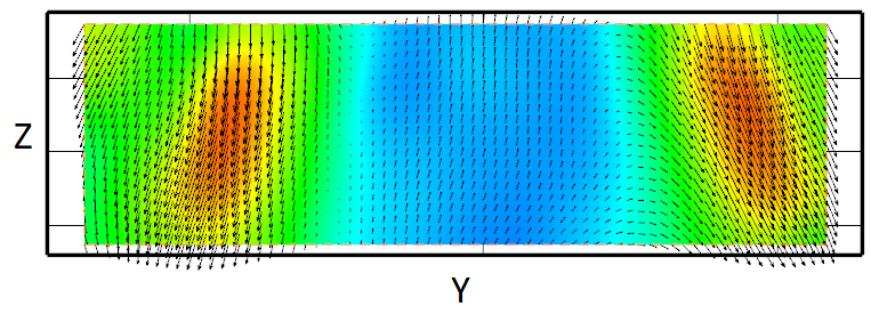

Figure 6 YZ slice showing ensemble average velocity vectors from the captured tomographic PIV data $(\mathrm{X}=\mathbf{0})$ 
ASME Journal of Engineering for Gas Turbines and Power

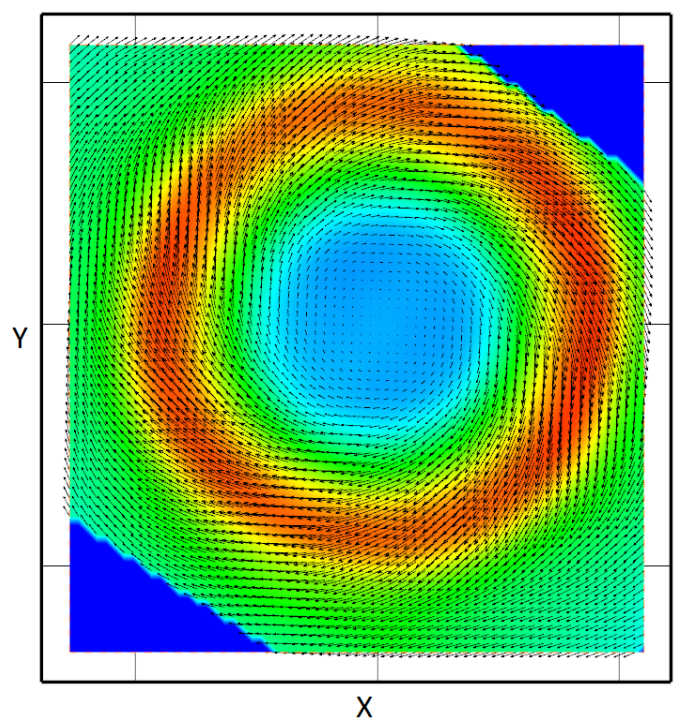

Figure 7 XY slice showing ensemble average velocity vectors from the captured tomographic PIV data $(\mathrm{Z}=\mathbf{0 . 2 5})$ 
ASME Journal of Engineering for Gas Turbines and Power

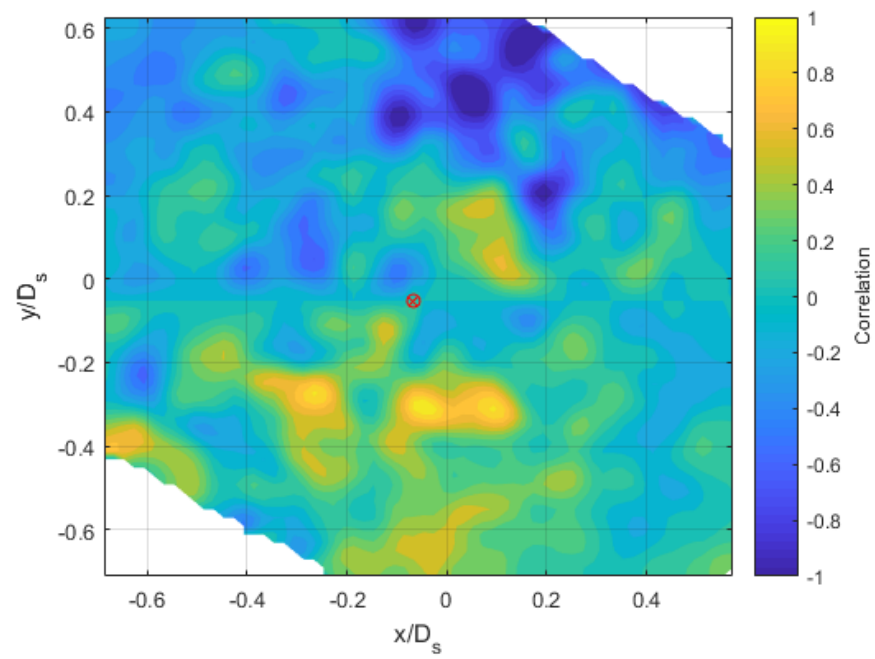

Figure 8 Example of correlation matrix for $U_{x}$ for the indicated sensor location. Correlation is normalized according to the maxima in the contour. 
ASME Journal of Engineering for Gas Turbines and Power

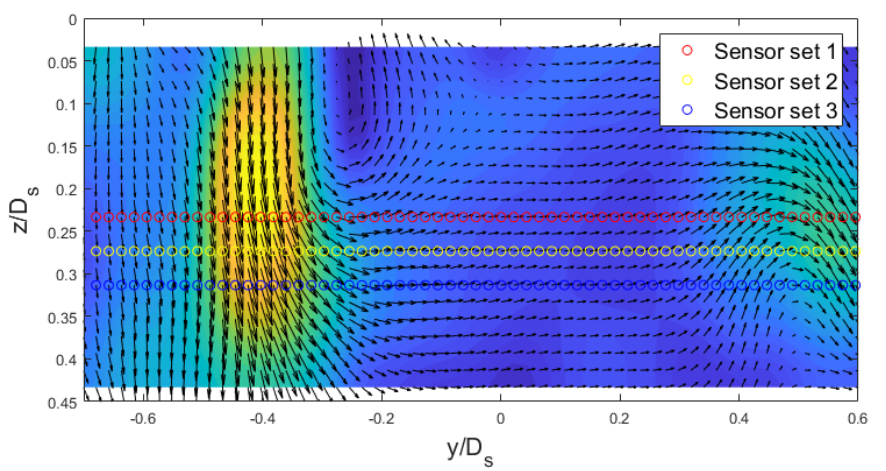

Figure 9 Example of instantaneous master plane with 3 example sensor sets highlighted. Contour shows velocity magnitude and in-plane vectors are displayed 
ASME Journal of Engineering for Gas Turbines and Power

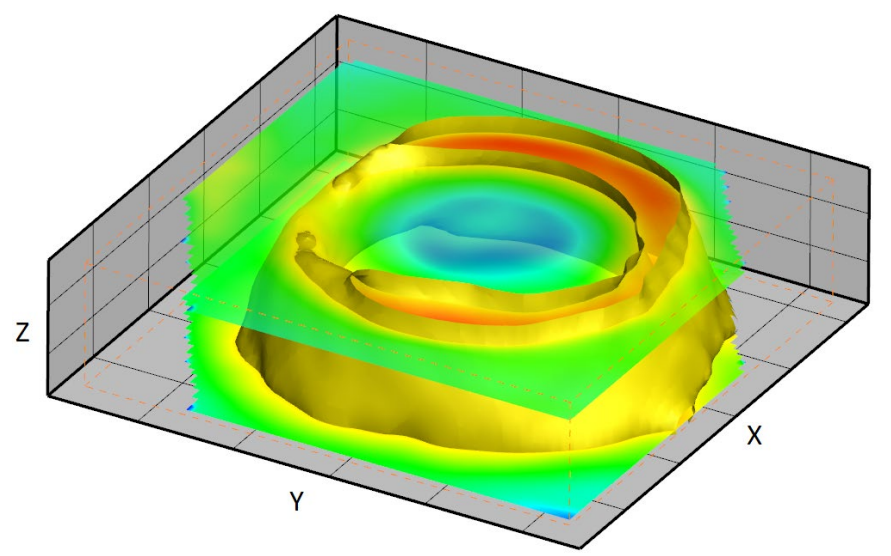

Figure 10 Ensemble mean of estimated velocity fields. Contours show velocity magnitude. Obtained from master fields with 60 POD mode reconstruction. 
ASME Journal of Engineering for Gas Turbines and Power

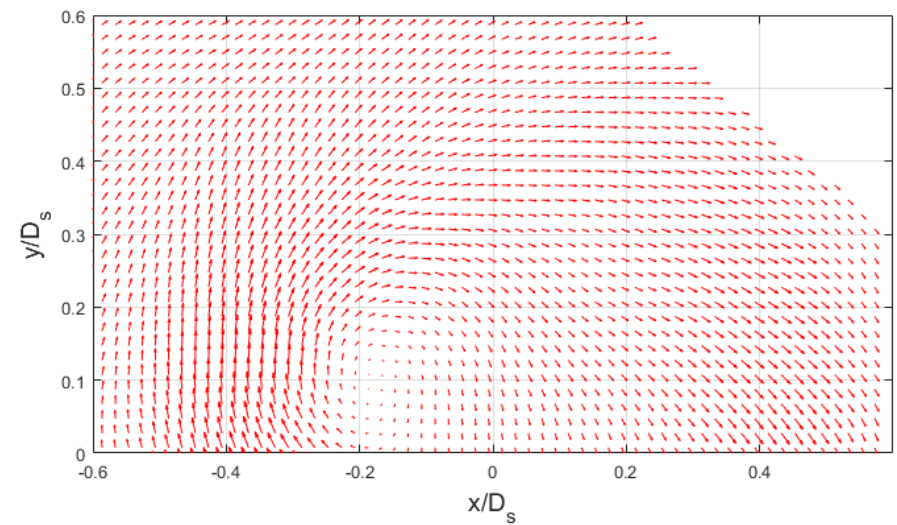

(a) Example extract of estimated velocity field, generated using 60 POD spatial modes

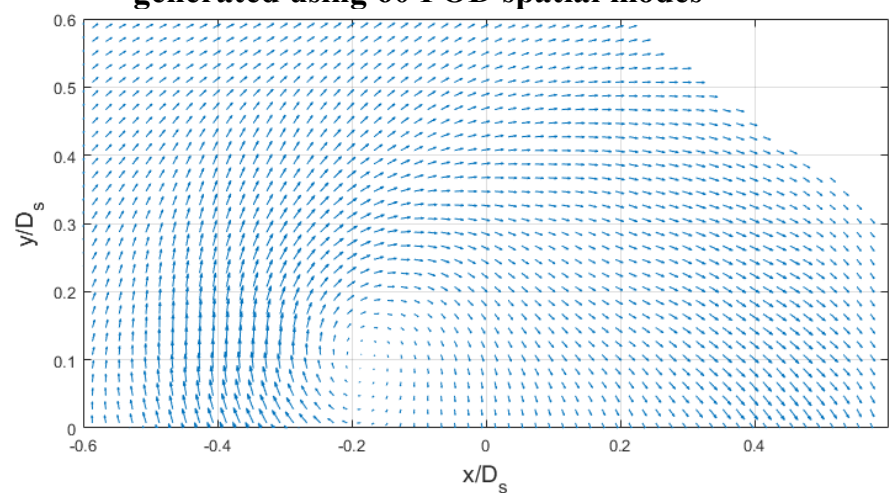

(b) Extract of the same plane and time instance taken from the validation PIV data set

Figure 11 Comparison of estimated velocity and validation velocity measurements for the same time instance (validation data set, $\mathbf{t}=\mathbf{1 0 1 0}$ ) 


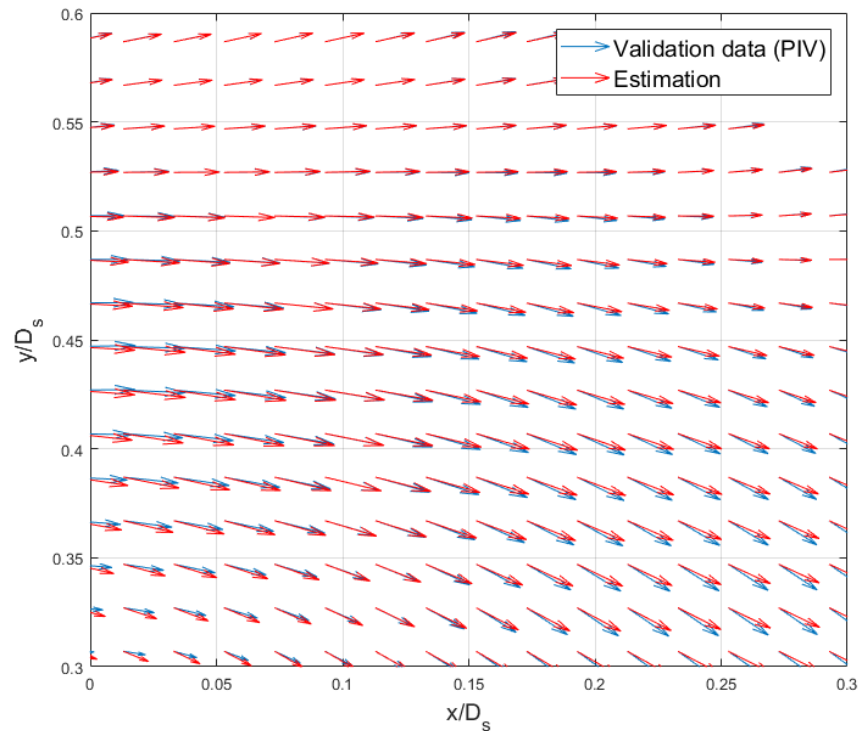

Figure 12 Comparison between validation data and estimated field (master plane pre-filtered, 70modes) 
ASME Journal of Engineering for Gas Turbines and Power

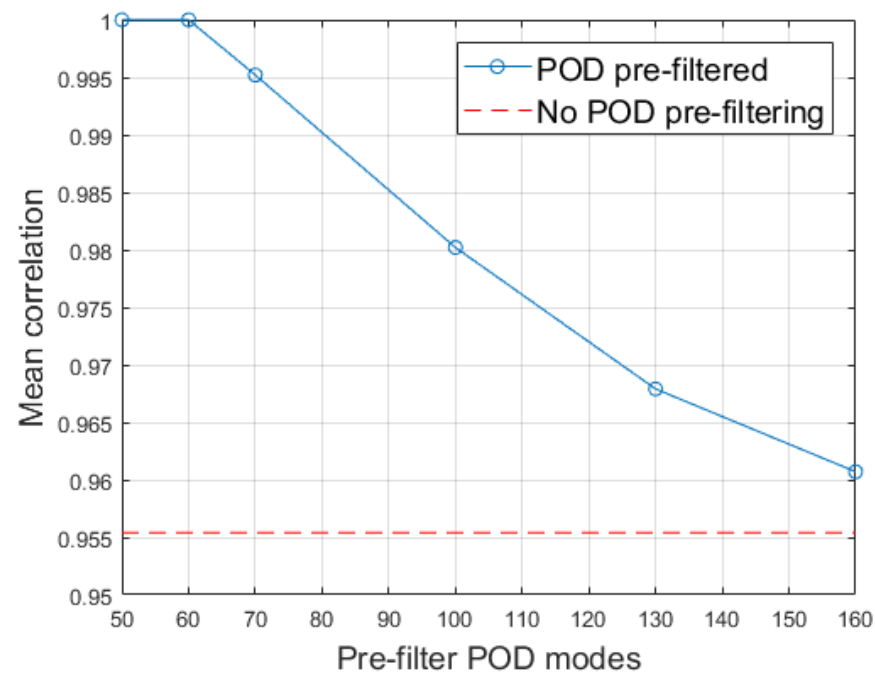

Figure 13 Mean correlation of estimated velocity and validation velocity for a range of pre-filtered and non-pre-filtered conditions 


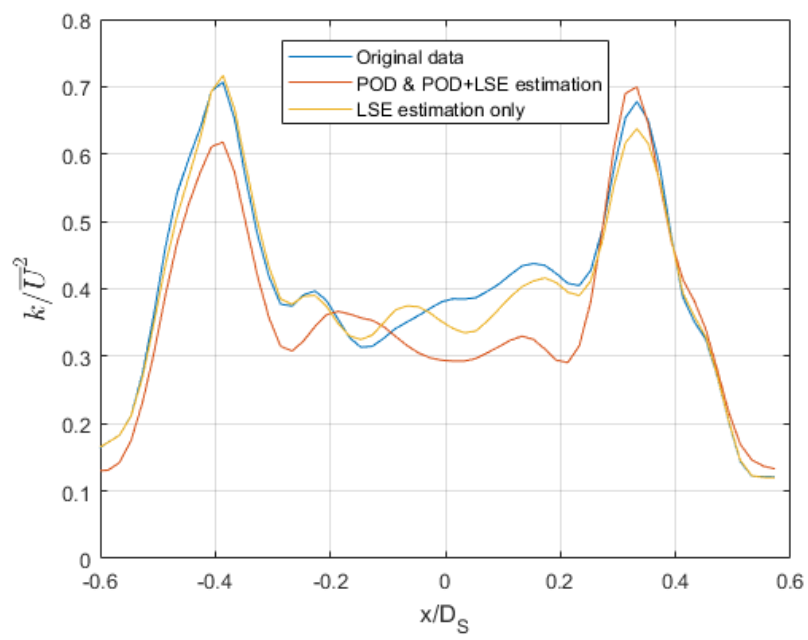

(a) Near field, $y / D_{s}=0.03, z / D_{s}=0.02$

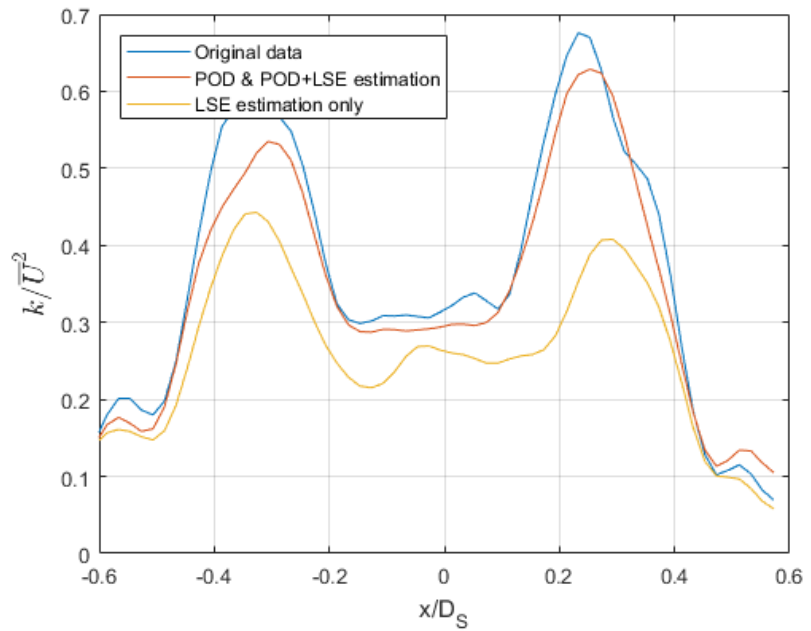

(b) Far field, $y / D_{s}=0.27, z / D_{s}=0.02$

Figure 14. Profiles of turbulent kinetic energy on a line parallel to the line of sensors. (intersection line at, $y / D_{s}=0.00$, $\mathrm{z} / \mathrm{D}_{\mathrm{s}}=0.02$ ) 